\title{
A Unified Probabilistic Framework for Volcanic Hazard and Eruption Forecasting
}

\author{
Warner Marzocchi ${ }^{1}$, Jacopo Selva ${ }^{2}$, Thomas H. Jordan ${ }^{3}$ \\ ${ }^{1}$ Department of Earth, Environmental, and Resources Sciences, University of Naples, Federico II, Complesso di Monte \\ 5 Sant'Angelo, Via Cupa Nuova Cintia, 21 - 80126 Napoli, Italy \\ 2 Istituto Nazionale di Geofisica e Vulcanologia, Via Donato Creti 12, 40128 Bologna, Italy \\ ${ }^{3}$ Department of Earth Sciences Southern California Earthquake Center, University of Southern California, Los Angeles, \\ California 90089
}

Correspondence to: Warner Marzocchi (warner.marzocchi@unina.it)

\begin{abstract}
The main purpose of this article is to emphasize the importance of clarifying the probabilistic framework adopted for volcanic hazard and eruption forecasting. Eruption forecasting and volcanic hazard analysis seeks to quantify the deep uncertainties that pervade the modeling of pre-, sin- and post-eruptive processes. These uncertainties can be differentiated into three fundamental types: (1) the natural variability of volcanic systems, usually represented as stochastic processes with

15 parameterized distributions (aleatory variability); (2) the uncertainty in our knowledge of how volcanic systems operate and evolve, often represented as subjective probabilities based on expert opinion (epistemic uncertainty); and (3) the possibility that our forecasts are wrong owing to behaviors of volcanic processes about which we are completely ignorant and, hence, cannot quantify in terms of probabilities (ontological error). Here we put forward a probabilistic framework for hazard analysis recently proposed by Marzocchi \& Jordan (2014), which unifies the treatment of all three types of uncertainty.

20 Within this framework, an eruption forecasting or a volcanic hazard model is said to be complete only if it (a) fully characterizes the epistemic uncertainties in the model's representation of aleatory variability and (b) can be unconditionally tested (in principle) against observations to identify ontological errors. Unconditional testability, which is the key to model validation, hinges on an experimental concept that characterizes hazard events in terms of exchangeable data sequences with well-defined frequencies. We illustrate the application of this unified probabilistic framework by describing experimental

25 concepts for the forecasting of tephra fall from Campi Flegrei. Eventually, this example may serve as a guide for the application of the same probabilistic framework to other natural hazards.
\end{abstract}

\section{Introduction}

Hazards associated with major eruptions and their consequences are highly uncertain owing to the stochastic behaviors of volcanic systems (aleatory variability) as well as our lack of knowledge about these behaviors (epistemic uncertainty).

30 Because such complexity hampers the deterministic prediction of hazards, our goal is to describe them probabilistically (Sparks, 2003; Marzocchi and Bebbington, 2012; Poland and Anderson, 2020). Here we use the term probabilistic volcanic 
hazard analysis (PVHA) to indicate the probabilistic forecast of any volcanological event of interest (eruption occurrence, ash fall loading, arrival of a pyroclastic flow, etc).

PVHA outcome may be generally described by the exceedance probability of a positive random variable $X$ (Martin et al., 2004, Selva et al., 2018; Rougier and Beven, 2013; Sandri et al., 2016; Bear-Crozier et al., 2016)

$f(x)=\operatorname{Pr}(X>x \mid \boldsymbol{H}), \quad x \in(0, \infty)$

where $\boldsymbol{H}$ is the PVHA model used, $f(x)$ is called the hazard curve (not to be confused with the hazard function commonly used to describe failure rate; from a statistical point of view $f(x)$ is a survival function). $X$ is the (continuous or discrete) hazard intensity of interest in one specific time interval, for example, the tephra fall loading in one specific site, the dynamic pressure of a pyroclastic flow in one sector of the volcano, or the occurrence of an eruption, etc. Ideally, since we never know the true hazard curve, we may include an additional level of uncertainty considering a set of PVHA models; in this case, we have $\left\{f_{i}(x), \pi_{i}\right\}(i=1, \ldots, N)$, where $\pi_{i}$ is the weight of the $i^{\text {th }}$ PVHA model $H_{i}, f_{i}(x) \equiv f\left(x \mid H_{i}\right)$ (Rougier and Beven 2013).

The way in which we use the set of hazard curves $f_{i}(x)$ to estimate PVHA depends inherently on the probabilistic

45 framework adopted. The importance of establishing the probabilistic framework adopted to calculate PVHA cannot be overstated, because such a framework implicitly defines the type of uncertainties, the meaning of probability, and eventually the possibility to validate (at least in principle) the PVHA model. Indeed, the qualitative difference between the objective probabilities of what we can see and count and the subjective probabilities of what we think we know has been recognized for 300 years, separating, for example, the frequentist and Bayesian approaches to probabilistic inference (Hacking 1965).

50 Despite recent attempts to unify these two approaches (e.g., Box 1980; Rubin 1984; Bayarri and Berger 2004; Berger, 2004; Gelman and Shalizi 2013), controversy remains over how aleatory variability can be cleanly separated from epistemic uncertainty and whether such a dichotomy is actually useful in hazard analysis. For example, in the Bayesian view adopted by many researchers, the only type of uncertainty is epistemic (e.g., NRC, 1997; Bedford and Cooke, 2001; Lindley, 2000; Jaynes, 2003), whereas in the traditional frequentist framework, the only type of uncertainty is aleatory (Hacking, 1965).

55 Actually, most practitioners recognize that distinguishing between different types of uncertainties can be useful in the interpretation of hazard estimates (e.g., Abrahamson and Bommer 2005; Rougier 2013), but the confusion surrounding the topic is evident in the wide variety of schemes proposed for classifying uncertainties: shallow and deep (Stein and Stein, 2013), intra- and inter-model, external and internal (Rougier and Beven 2013), inter- and intra-model (Selva et al., 2013), value and structural uncertainty (e.g., Solomon et al., 2007), quantified measure of uncertainty and confidence on the validity 60 of a finding (IPCC, 2013), model parameters and initial/boundary conditions, and many others (Reilly et al. 2021). It has been unclear whether these classifications are profound categories that must be reflected in the probabilistic framework or merely convenient, model-based divisions (e.g., NRC, 1997; Rougier and Beven, 2013).

The subject of this paper is to underline the importance of the probabilistic framework in PVHA and its practical implications. This framework must be able to (1) establish a coherent and clear hierarchy of different kinds of uncertainty 
65 (aleatory variability, epistemic uncertainty and ontological error), (2) assimilate subjective expert judgment into probabilistic models, and (3) unconditionally test complete probabilistic models against data. In the next sections, through a toy example and then an application to Campi Flegrei tephra-fall PVHA, we describe the application to PVHA of this novel unified probabilistic framework developed by Marzocchi and Jordan (2014, 2017, 2018), which satisfies these requirements.

\section{A tutorial example to describe a unified probabilistic framework for PVHA}

70 We consider the case in which $f(x)$ is the annual probability to exceed a specific tephra fall threshold $x$ in one site around a volcano (the probability of exceedance, or PoE). The results can be generalized in a straightforward way to other time windows and other types of volcanic threats, as well as to eruption forecasting.

We collect the sequence of annual observations for a particular value of the tephra fall loading $x_{0}$, which we denote by the binary variable $e_{i}$. That is, $e_{i}=0$ when there is no exceedance in the $i^{\text {th }}$ year $\left(x<x_{0}\right)$, and $e_{i}=1$ when there is $\left(x \geq x_{0}\right)$.

75 Then we judge $e_{i}$ as stochastically exchangeable, i.e., with joint probability distributions invariant to data ordering when conditioned on a set of explanatory variables (Draper et al. 1993). The De Finetti theorem (1974) states that exchangeable events coming from different and unknown distributions (such as a set of "non-repeatable" events) can be modeled as identical and independently distributed random variables with a well-defined frequency of occurrence, $\hat{\phi}$. The frequentist interpretation applied to a set of exchangeable events and the use of the Bayesian mathematical apparatus to handle

80 uncertainties of different kind is the reason for use the term "unified" to characterize the probabilistic framework described in this paper.

In our example, $\hat{\phi}=f\left(x_{0}\right)$, and the unknown frequency $\hat{\phi}$ of the exchangeable sequence $e_{i}$ is the aleatory variability and the target of PVHA. The unknown true aleatory variability is often estimated by different models, $\left\{\phi_{i}, \pi_{i}\right\}$, where $\pi_{i}$ is the weight of the $i^{\text {th }}$ PVHA model $f_{i}(x)$, and $\phi_{i}=f_{i}\left(x_{0}\right)$. From the set $\left\{\phi_{i}, \pi_{i}\right\}$ we estimate the PoE distribution $p(\phi)$ (hereafter

85 we use $p(\cdot)$ and $P(\cdot)$ to indicate the probability density function and the cumulative distribution, respectively). This procedure is non-unique, it contains a degree of subjectivity, but it is unavoidable; for example, choosing only one estimation $\phi_{i}$, or the weighted average assumes that $p(\phi)$ follows a Dirac distribution centered to this value. We call $p(\phi)$ "Extended Experts' Distribution (EED)", which describes the full PVHA (Marzocchi and Jordan, 2014).

The EED allows model validation. Specifically, we can now define an ontological null hypothesis, which states that the true 90 aleatory representation of future occurrence of natural events - the data generating process - mimics a sample from the probability distribution of aleatory representations that describe the model's epistemic uncertainty. According to the ontological null hypothesis, the true unknown frequency $\hat{\phi}$ of the experimental concept cannot be distinguished from a realization of the EED, i.e., $\hat{\phi} \sim p(\phi)$. If the data are inconsistent with the EED, the ontological null hypothesis can be rejected, which identifies the existence of an ontological error (Marzocchi and Jordan, 2014). In other words, the "known 
unknowns" do not necessarily completely characterize the uncertainties, presumably due to some effect not included in the EED—an "unknown unknown".

In practice, collecting sufficient data for this kind of model validation is feasible for specific sites surrounding active volcanoes with a high frequency of eruptions. For a specific site near a high-risk volcano with a low eruptive frequency, the data are usually insufficient for formal ontological testing. In probabilistic seismic hazard analysis (PSHA), the problem is overcome trading time with space, i.e., considering many sites simultaneously one or more time windows. This approach requires that exceedances recorded at different sites can be considered statistically independent; although this may become attainable in PSHA under some specific considerations, this clearly does not hold for sites surroundings a single volcano. In volcanology, trading time with space is useful in validating models for global PVHA (e.g., Jenkins et al., 2015), which consider the eruptive activity of all volcanoes of a specific type. In this case, it is possible to select sites that are far enough to consider the observed exceedances conditionally independent one from each other, and the exchangeable sequence $e_{i}(i=$ $1, \ldots, N)$ is given by the annual exceedances observed at different $N$ sites.

The exchangeability judgment can be generalized beyond the stationarity of the process (implicit in our example) to more complex situations. For example, we may distinguish exceedance events in years when one large earthquake occurred nearby the volcano from those in years when no earthquakes occurred. The data-generating process provides two sequences,

$110\left\{e_{i}^{(1)}: i=1, \ldots, N_{1}\right\}$ for the $N_{1}$ years in which a large earthquake occurred, and $\left\{e_{i}^{(0)}: i=1, \ldots, N_{0}\right\}$ for the $N_{0}$ years in which no large earthquakes occurred. Both are judged to be Bernoulli, and they are observed to sum to $\mathrm{k}_{0}$ and $\mathrm{k}_{1}$ respectively. If the occurrence of an eruption is more likely when a large earthquake occurred nearby, then the expected frequency of $\hat{\phi}^{(1)}=k_{1} / N_{1}$ might be greater than that of $\hat{\phi}^{(0)}=k_{0} / N_{0}$. As this example makes clear, it is neither the aleatory variability intrinsic to the model that matters in testing, nor to the true process of the world, but rather the aleatory

115 variability is defined by the exchangeability judgments of the experimental concept. In other words, aleatory variability is an observable behavior of the data-generating process - nature itself - conditioned by the experimental concept to have welldefined frequencies (Marzocchi and Jordan, 2014).

\section{Accounting for epistemic uncertainty and aleatory variability: the unified framework applied to the tephra fall PVHA at Campi Flegrei}

120 In this section we apply the probabilistic framework outlined in section 2 to the tephra fall PVHA at Campi Flegrei. Although the low eruption frequency of this volcano makes model validation unrealistic in the human being time frame, the probabilistic framework has the remarkable advantage to provide a full description of the PVHA accounting for all uncertainties; this may be of particular importance for users because, for example, they can immediately appreciate the level of uncertainty over the probabilistic assessment made by volcanologists. 
125 Most (if not all) of the studies available for tephra fall PVHA at Campi Flegrei are based on Event Trees (ET; see Newhall and Hoblitt, 2002; Marzocchi et al., 2004; 2008; 2010; Marti et al., 2008; Sobradelo and Marti, 2010). The ET is a popular tree graph representation of events in which individual branches at each node point to different possible events, states, or conditions through increasingly specific subsequent events (intermediate outcomes) to final outcomes; in this way, an ET shows all relevant possible outcomes of volcanic unrest at progressively higher degrees of detail. The probability of each outcome is calculated combining the conditional probability of each branch belonging to the path from the first node to the final outcome through classical probability theorems. By construction, ET is meant to describe only the intrinsic variability of the process (aleatory variability) and not the epistemic uncertainty, hence it may produce only one single probabilistic assessment $f(x)$. The ET has been generalized to account for uncertainties of different kind replacing the probability at each node with a distribution of probability (Bayesian Even Tree, BET; Marzocchi et al., 2004; 2008; 2010; Neri et al., 2008;

135 Sobradelo and Marti, 2010), which aims at accounting for experts' judgment, different conceptual or physical models, data from analog volcanoes, as well as data from the target or analog volcanoes (Marzocchi et al., 2004; Tierz et al., 2019).

The BET approach fits quite well the unified probabilistic framework that we advocate in this paper. If we consider an experimental concept given by an exchangeable sequence $e_{i}$ of annual tephra fall exceedances, the outcome of the BET code may be seen as an EED, i.e., the distribution of probability $p(\phi)$ mimics where the true unknown frequency of the exchangeable sequence may be. In the first paper that describes BET (Marzocchi et al., 2004), the use of a distribution of probability at each node was generically advocated to account for the aleatory variability and epistemic uncertainty, using the loose definition of irreducible randomness and limited knowledge of the process, respectively. However, relating these quantities to the true process of the world, the separation between them remains elusive, because, as far as our knowledge of the process increases, all uncertainties become necessarily epistemic. This paper provides a formal probabilistic background

145 to justify the BET feature of considering the probability at each node as a distribution $(p(\phi))$ instead of a single number; at each node of the event tree, the central value of the distribution is the best guess of the (unknown) long-term frequency of the experimental concept for that specific node (aleatory variability), and the dispersion mimics the uncertainty over this unknown frequency.

The BET approach has been widely investigated for tephra fall PVHA at Campi Flegrei, adopting different choices,

150 hypotheses, and models (Selva et al., 2010; 2018; Sandri et al., 2016). For instance, Selva et al (2018) show the outcomes of five different BET configurations (Figure 1) for one specific site inside the caldera (Figure 2), which differ in the implementation of the tephra fall dispersion model (aggregation and granulometry). In Figure 3 we show the PoE distribution $p(\phi)$ of each configuration for the tephra loading threshold of $300 \mathrm{~kg} / \mathrm{m}^{2}$. Each one of the five EED distributions allows for a formal testing of the ontological hypothesis.

155 When all EEDs are significantly overlapping (as for many points inside the Campi Flegrei caldera), it means that each BET configuration describes the epistemic uncertainty in a consistent manner. Instead, for the site in Figure 2, we infer that inconsistent BET outcomes (Figure 3) may be due to an underestimation of the epistemic uncertainty in each EED. For this 
reason, in the example of Figure 3 we consider only the weighted average of each EEDs and then we build a new EED which describes more satisfactorily the overall epistemic uncertainty given by the five BET configurations. This is somehow equivalent to the case of using alternative implementations of the classical ET (Newhall and Pallister, 2015), which produces a set of hazard curves like in the upper panels of Figure 1. In the specific case of Figure 3, the reason for which the epistemic uncertainty is underestimated in each EED may be due to the BET set up and/or to limitations of BET model to handle some sources of epistemic uncertainty.

The way in which we can build a single EED from a set of point forecasts $\left\{\phi_{l}, \pi_{l}\right\}$ (lower panels of Figure 1), or from a set of inconsistent EEDs (Figure 3), is what we call ensemble modeling. The terms ensemble modeling and ensemble forecasts have been used in many disciplines in different ways since early seventies (e.g., Leith, 1974). The recent Nate Silver's book (Silver, 2012) gives a wide range of successful applications and uses of ensemble modeling. The common feature across all these different flavors of ensemble modeling/forecasts is the attempt to account for the aleatory variability and the epistemic uncertainty by merging the forecasts of different models or parametrization of the same model in a proper way.

170 Standard methods are available for the induction of the $\operatorname{EED} p(\phi)$ from the set $\left\{\phi_{i}, \pi_{i}\right\}$. Regardless the details of the procedure, we underline that the nonunique extrapolation of $\left\{\phi_{i}, \pi_{i}\right\}$ onto the continuous distribution $p(\phi)$ can contribute to ontological errors in the EED. Nonetheless, any reasonable procedure is not more subjective, and certainly less critical, than either not considering the epistemic uncertainty (for example, when using the weighted average $\bar{\phi}$ ), or assuming that the set of forecasts explore completely and exhaustively the epistemic uncertainty. In the first case, it is assumed that $p(\phi) \equiv$

$175 \delta(\Phi-\bar{\phi})$ is the Dirac distribution; in the latter case, it is assumed that the proper distribution for the epistemic uncertainty is exhaustively described by the set of forecasts, i.e., one of the forecasts must provide the true value (e.g., excluding the possibility that the true value is inside possible "empty space" between forecasts, or above/below the largest/smallest; Marzocchi et al., 2015).

Although ensemble modeling does not prescribe any specific procedure to estimate $p(\phi)$, in statistics random variables

180 bounded in the range [0,1] are often modeled by means of the Beta distribution (Gelman et al., 2003). Hence, we assume that $\Phi \sim \operatorname{Beta}(\alpha, \beta)$, where the parameters $\alpha$ and $\beta$ are related to the weighted average $E(\phi)$ and variance $\operatorname{var}(\phi)$ of $\left\{\phi_{i}, \pi_{i}\right\}$ through:

$E(\phi)=\frac{\alpha}{(\alpha+\beta)}$

and

$185 \operatorname{var}(\phi)=\frac{\alpha \beta}{(\alpha+\beta)^{2}(\alpha+\beta+1)}$

Inverting equations 2 and 3, we can get the parameters of the Beta distribution $p(\phi)$, which describes the ontological hypothesis. Using the weights reported in Figure 1, we get the Beta distribution reported in Figure 4. As expected, this 
global EED is wider than each single EED of the BET configurations reported in Figure 3, and it aims at describing more realistically the complete uncertainty in PVHA. Although the choice of the Beta distribution is subjective, we advocate the use of a unimodal distribution (the Beta distribution is almost always unimodal), which describes more realistically, in most cases, the epistemic uncertainty over the true (unknown) frequency. (See the discussion of Figure 6 in Marzocchi et al., 2015.)

\section{Discussion and conclusions}

In this paper we have described a unified probabilistic framework which allows volcanologists to provide a complete description of PVHA, to define a clear taxonomy of uncertainties (aleatory variability, epistemic uncertainty, and ontological errors), and to account for experts' judgments preserving the possibility to unconditionally test PVHA against data, at least for high-frequency erupting volcanoes, or for global forecasting models. Although in this paper we focus entirely on PVHA, we think that this approach may potentially inspire other scientists working on different natural hazards.

One remarkable and distinctive feature of this probabilistic framework is that the mathematical description of PVHA is given

200 by a distribution of probability (see, e.g., Marzocchi et al., 2004; 2008; 2010; Neri et al., 2008; Sobradelo and Marti, 2010; Bevilacqua et al., 2015), or, equivalently, through a bunch of hazard curves $f_{i}(x)(i=1, \ldots, N)$ (Rougier and Beven, 2013). The use of a distribution of probability instead of single numbers mark the main difference with probabilistic frameworks that are more commonly used in PVHA (Marzocchi and Bebbington, 2012), i.e., the frequentist (e.g., Bebbington, 2010; Deligne et al., 2010), and subjective Bayesian (Aspinall et al., 2003). Although these probabilistic frameworks are both

205 legitimate because they are coherent with the Kolmogorov's axioms, they cannot provide a complete description of PVHA, because they cannot unambiguously distinguish and handle properly uncertainties of different kind, which are likely pervasive in natural systems (Marzocchi and Jordan, 2017; 2018).

The importance of making explicit the probabilistic framework in PVHA cannot be overstated. In the past, loose definitions of the probabilistic framework adopted nurtured severe critics to natural hazard analysis (see, e.g., Castanos and Lomnitz,

210 2002; Mulargia et al., 2017). For example, a vague definition of the nature of uncertainties and the role of subjective judgments brought some scientists to assert that (Stark, 2017) "what appears to be impressive 'science' is in fact an artificial amplification of the opinions and ad hoc choices built into the model, which has a heuristic basis rather than a tested (or even testable) scientific basis." This criticism is implicitly rooted in the (false) syllogism: science is objective, natural hazard analysis relies on subjective experts' judgment, hence natural hazard analysis is not science.

215 The unified probabilistic framework proposed here emphasizes the importance of model validation (at least in principle) as cornerstone of science; pure objectivity is a myth even in science and the presence of unavoidable subjectivity in PVHA cannot be used to dismiss its scientific nature. In an extreme case, experts' can behave like "models" expressing their subjective measure of the frequency of one defined experimental concept; mutatis mutandis, the same applies to the famous 
case of farmers who subjectively guess the weight of an ox (Galton, 1907), whose similarities and differences with natural

hazard analysis has been carefully discussed in Marzocchi and Jordan (2014).

Besides the scientific aspects, the use of a PoE distribution has remarkable practical merits, because it shows to the decision makers both our best guess and the associated uncertainty. In plain words, if two PVHA have the same average, but with quite different variance, decision makers can immediately appreciate the difference in the scientific assessment.

As a final consideration, owing to the social implications, we think that only adopting a clear probabilistic framework to get a complete PVHA is the best way to defend probabilistic assessments against future scrutiny and criticism and to use these assessments in the most profitable way.

Author contribution: All authors contributed, at different levels, to the conceptualization of the research. WM and JS carried out the formal analysis and numerical investigation. WM wrote the first draft of the paper with contributions from all the other authors. All authors reviewed and edited the final version.

\section{Acknowledgments: TO BE WRITTEN}

\section{References}

Miller, B. B. and Carter, C.: The test article, J. Sci. Res., 12, 135-147, doi:10.1234/56789, 2015.

Smith, A. A., Carter, C., and Miller, B. B.: More test articles, J. Adv. Res., 35, 13-28, doi:10.2345/67890, 2014.

Abrahamson, N. A., and Bommer, J. J.: Probability and uncertainty in seismic hazard analysis, Earthq. Spectra, 21, 603-607, 2015.

Aspinall, W.P., Woo, G., Voight, B., and Baxter, P.: Evidence-based volcanology: application to eruption crises, J. Volcanol. Geoth. Res., 128, 273-285, 2003.

Bayarri, M.J., and Berger, J.: P-values for composite null models, J. Am. Stat. Assoc., 95, 1127-1142, 2000.

Bear-Crozier, N., Miller, V., Newey, V., Horspool, N., and Weber, R.: Probabilistic Volcanic Ash Hazard Analysis (PVAHA) I: development of the VAPAH tool for emulating multi-scale volcanic ash fall analysis. J. App. Volc., 5, 3, 2016.

Bedford, T., and Cooke, R.: Probabilistic risk analysis: foundations and methods, Cambridge University Press, 2001.

Berger, J.: The case for objective Bayesian analysis, Bayesian Anal., 1, 1-17, 2004.

Bevilacqua, A., Isaia, R., Neri, A., Vitale, S., Aspinall, W.P., Bisson, M., Flandoli, F., Baxter, P. J., Bertagnini, A., Esposti Ongaro, T., Iannuzzi, E., Pistolesi, M., and Rosi, M.: Quantifying volcanic hazard at Campi Flegrei caldera (Italy) with uncertainty assessment:1. Vent opening maps, J. Geophys. Res. Solid Earth, 120, 2309-2329, 2015.

Box, G.E.P.: Sampling and Bayes inference in scientific modelling and robustness, Roy. Statist. Soc. Ser. A 143, 383-430. 1980.

Castaños, H., and Lomnitz, C.: PSHA: Is it science? Eng Geol, 66, 315-317, 2002. 
de Finetti, B.: Theory of Probability: A Critical Introductory Treatment, John Wiley and Sons, London, 1974.

Deligne, N. I., Coles, S. G., and Sparks, R. S. J.: Recurrence rates of large explosive volcanic eruptions. J. Geophys. Res. Solid Earth, 115, B06203, 2010.

Draper, D., Hodges, J., Mallows, C., and Pregibon, D.: Exchangeability and data analysis (with discussion). J. Roy. Statist. Soc, Ser. A, 156, 9-37, 1993.

Galton, F.: Vox populi, Nature, 75, 450-451, 1907.

255 Gelman, A., Carlin, J. B., Stern, H. S., and Rubin, D. B.: Bayesian Data Analysis, second edition, Chapman and Hall, London, 2003.

Gelman, A., and Shalizi C. R.: Philosophy and the practice of Bayesian statistics. Br. J. Math. Stat. Psychol., 66, 8-38, 2013.

Hacking, I.: The Logic of Statistical Inference, Cambridge University Press, Cambridge, 1965.

IPCC: Climate Change 2013: The Physical Science Basis. Contribution of Working Group I to the Fifth Assessment Report of the Intergovernmental Panel on Climate Change [Stocker, T.F., D. Qin, G.-K. Plattner, M. Tignor, S.K. Allen, J. Boschung, A. Nauels, Y. Xia, V. Bex and P.M. Midgley, 2013.

(eds.)]. Cambridge University Press, Cambridge, United Kingdom and New York, NY, USA, 1535 pp.

Jaynes, E.T.: Probability theory: the logic of Science, Cambridge Univ. Press, NewYork, 2003.

265 Jenkins, S. F., Wilson, T. M., Magill, C., Miller, V., Stewart, C., Blong, R., Marzocchi, W., Boulton, M., Bonadonna, C., and Costa, A.: Volcanic ash fall hazard and risk. In: Susan Loughlin, Steve Sparks, Sarah Brown, Susanna Jenkins, Charlotte Vye-Brown (Eds.), Global Volcanic Hazards and Risk, pp. 173-222, 2015.

Leith, C.E.: Theoretical Skill of Monte Carlo Forecasts, Monthly Weather Rev., 102, 409-418, 1974.

Lindley, D.V.: The philosophy of statistics, Statistician 49, 293-337, 2000.

270 Marti, J., Aspinall, W. P., Sobradelo, R., Felpeto, A., Geyer, A., Ortiz, R., Baxter, P., Cole, P., Pacheco, J., Blanco, M. J., and Lopez, C.: A long-term volcanic hazard event tree for Teide-Pico Viejo stratovolcanoes (Tenerife, Canary Islands). J. Volcanol. Geoth. Res., 178, 543-552, 2008.

Martin, A.J., Umeda, K., Connor, C. B., Weller, J. N., Zhao, D., and Takahashi, M.: Modeling long-term volcanic hazards through Bayesian inference: an example from the Tohoku volcanic arc, Japan. J. Geophys. Res., 109, B10208, 2004.

275 Marzocchi, W., Sandri, L., Gasparini, P., Newhall, C. N., and Boschi, E.: Quantifying probabilities of volcanic events: the example of volcanic hazard at Mount Vesuvius, J. Geophys. Res., 109, B11201, 2004.

Marzocchi, W., Sandri, L., and Selva, J.: BET_EF: a probabilistic tool for long- and short-term eruption forecasting, Bull. Volcanol., 70 (5), 623-632, 2008.

Marzocchi, W., Sandri, L., and Selva, J.: BET_VH: a probabilistic tool for long-term volcanic hazard assessment, Bull. 280 Volcanol., 72, 705-716, 2010.

Marzocchi, W., and Bebbington, M.: Probabilistic eruption forecasting at short and long time scales, Bull. Volcanol., 74, $1777-1805,2012$.

Marzocchi, W., and Jordan, T. H.: Testing for Ontological Errors in Probabilistic Forecasting Models of Natural Systems, Proc. Nat. Acad. Sci. 85, 955-959, 2014.

285 Marzocchi, W., Taroni, M., and Selva, J.: Accounting for epistemic uncertainty in PSHA: logic tree and ensemble modeling. Bull. Seismol. Soc. Am., 105(4), 2151-2159, 2015. 
Marzocchi, W., and Jordan, T. H.: A unified probabilistic framework for seismic hazard analysis, Bull. Seismol. Soc. Am., 107(6), 2738-2744, 2017.

Marzocchi, W., and Jordan, T. H.: Experimental concepts for testing probabilistic earthquake forecasting and seismic hazard models, Geophys. J. Int., 215, 780-798, 2018.

Mulargia, F., Stark, P. B., and Geller, R. J.: Why is probabilistic seismic hazard analysis (PSHA) still used? Phys. Earth Planet. Int., 264, 63-75, 2017.

Neri, A., Aspinall, W.P., Cioni, R., Bertagnini, A., Baxter, P. J., Zuccaro, G., Andronico, D., Barsotti, S., Cole, P. D., Esposti Ongaro, T., Hincks, T. K., Macedonio, G., Papale, P., Rosi, M., Santacroce, R., and Woo, G.: Developing an Event Tree for Probabilistic Hazard and Risk Assessment at Vesuvius, J. Volcan. Geoth. Res., 178, 397-415, 2008.

Newhall, C. G., and Hoblitt, R.: Constructing event trees for volcanic crises. Bull. Volcanol. 64, 3-20, 2002.

Newhall, C. G., and Pallister, J. S.: Using Multiple Data Sets to Populate Probabilistic Volcanic Event Trees, in: Volcanic Hazards, Risks and Disasters, Ed(s): J. F. Shroder, P. Papale, Elsevier, 203-232, 2015.

NRC, National Research Council Panel on Seismic Hazard Evaluation: Review of Recommendations for Probabilistic 300 Seismic Hazard Analysis: Guidance on Uncertainty and Use of Experts, National Academy of Sciences, Washington, D.C., 84 pp., ISBN:0-309-56207-4, 1997.

Poland, M. P., and Anderson, K. R.: Partly cloudy with a chance of lava flows: Forecasting volcanic eruptions in the twentyfirst century, J. Geophys. Res. Solid Earth, 125, e2018JB016974, 2020.

Reilly, A. C., Baroud, H., Flage, R., and Gerst, M. D.: Sources of uncertainty in interdependent infrastructure and their implications, Reliability Engineering \& System Safety, 213, 107756, doi:10.1016/j.ress.2021.107756, 2021.

Rougier, J. C.: Quantifying hazard losses. In: Risk and uncertainty assessment for natural hazards, eds. J. Rougier, S. Sparks, and L. Hill. Cambridge University press, Cambridge, 19-39, 2013.

Rougier, J. C., and Beven, K. J.: Model and data limitations: the sources and implications of epistemic uncertainty. In: Risk and uncertainty assessment for natural hazards, eds. J. Rougier, S. Sparks, and L. Hill. Cambridge University press, 310 Cambridge, 40-63, 2013.

Rubin, D.B.: Bayesian justifiable and relevant frequency calculations for the applied statistician, Ann Stat, 12, 1151-1172, 1984.

Sandri, L., Costa, A., Selva, J., Macedonio, G., Tonini, R., Folch, A., and Sulpizio, R.: Beyond eruptive scenarios: tephra fall from Neapolitan volcanoes, Sci. Rep., 6, 24271, 2016.

315 Selva, J., Costa, A., Marzocchi, W., and Sandri, L.: BET_VH: Exploring the infuence of natural uncertainties on long-term hazard from tephra fallout at Campi Flegrei (Italy), Bull. Volcanol., 72, 717-733, 2010.

Selva, J., Argyroudis, S., and Pitilakis, K.: Impact on loss/risk assessments of inter-model variability in vulnerability analysis, Natural Hazards 67, 723-746, 2013.

Selva, J., Costa, A., De Natale, G., Di Vito, M. A., Isaia, R., and Macedonio, G.: Sensitivity test and ensemble hazard assessment for tephra fallout at Campi Flegrei, Italy, J. Volcanol. Geotherm. Res., 351, 1-28, 2018.

Silver, N.: The Signal and the Noise: Why So Many Predictions Fail-but Some Don't, The Penguin Press, New York, New York, 2012.

Sobradelo, R., and Marti, J.: Bayesian event tree for long-term volcanic hazard assessment: application to Teide-Pico Viejo stratovolcanoes, Tenerife, Canary Islands, J. Geophys. Res., 115, B05206, 2010. 
https://doi.org/10.5194/nhess-2021-213

Preprint. Discussion started: 23 July 2021

(c) Author(s) 2021. CC BY 4.0 License.

(c) (1)

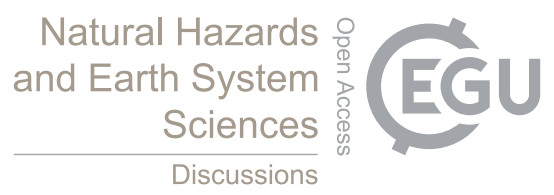

325 Solomon, S., Qin, D., Manning, M., Chen, Z., Marquis, M., Averyt, K. B., Tignor, M., and Miller, H. L. eds.: Climate change 2007: the physical science basis: contribution of Working Group I to the fourth assessment report of the intergovernmental panel on climate change. Cambridge and New York, Cambridge University Press, 2007.

Sparks, R.S.J.: Forecasting volcanic eruptions, Earth Plan. Sci. Lett., 210, 1-15, 2003.

Stark, P. B.: Pay no attention to the model behind the curtain, in Significant Digits: Responsible Use of Quantitative

330 Information, A. Saltelli and Â. Guimarães Pereira (Editors), Megaloceros Press, University of Bergen, Bergen, Germany, 2017.

Stein, S., and Stein, J. L.: Shallow versus deep uncertainties in natural hazard assessments. EOS, Tran. AGU, 94, 133-140, 2013.

Tierz, P., Loughlin, S. C., and Calder, E. S.: VOLCANS: an objective, structured and reproducible method for identifying 335 sets of analogue volcanoes, Bull. Volcan., 81, 76, 2019. 
https://doi.org/10.5194/nhess-2021-213

Preprint. Discussion started: 23 July 2021

(c) Author(s) 2021. CC BY 4.0 License.

(c) (1)
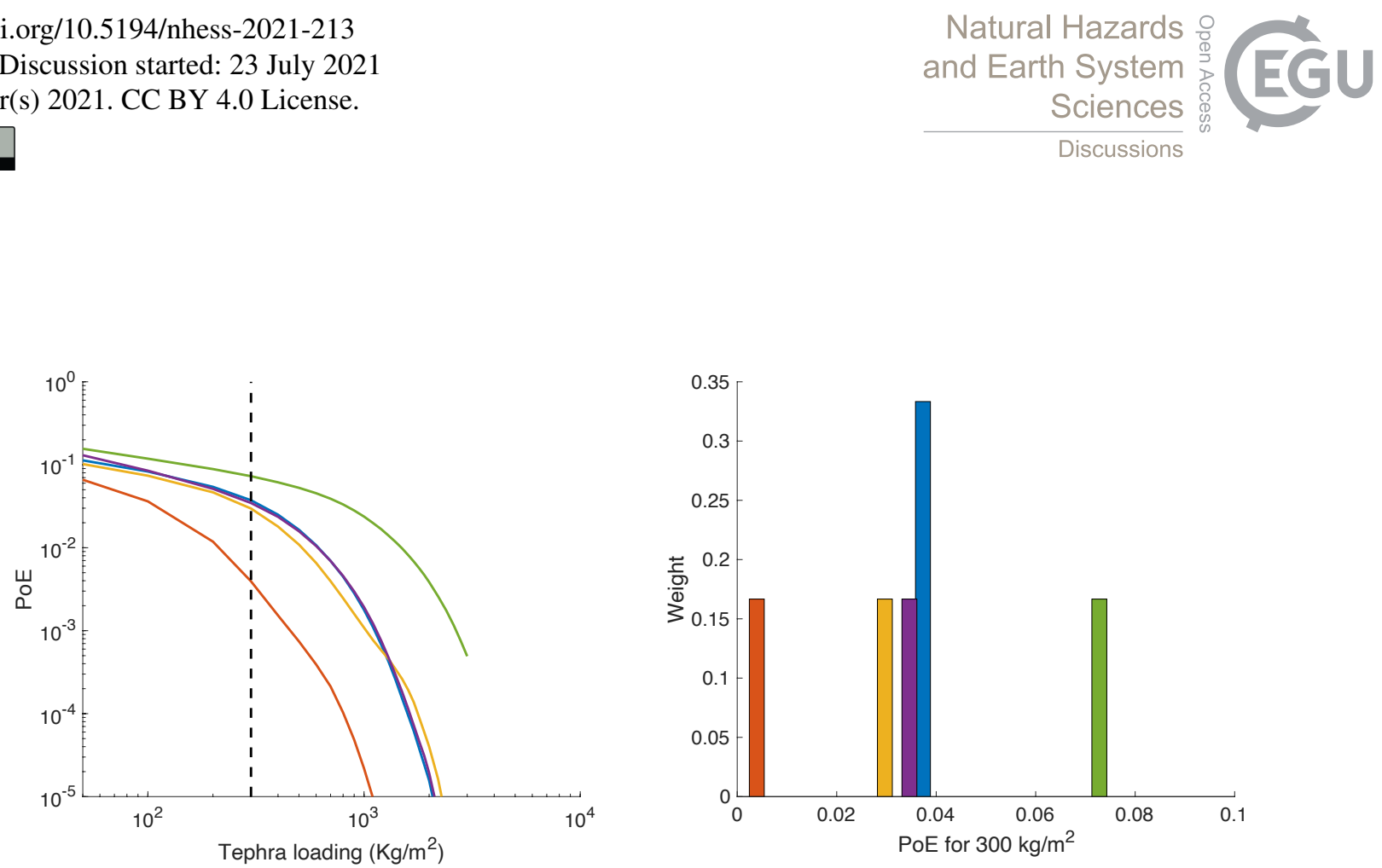

Figure 1: The left panel shows the mean tephra fall hazard curves relative to one point inside the Campi Flegrei (Figure 2) for each BET implementation described in Selva et al. (2018); colors indicate each implementation. The right panel shows the PoE relative to the tephra load threshold of $300 \mathrm{~kg} / \mathrm{m}^{2}$ (vertical dashed line in the upper panel), and the weight of each assessment. 


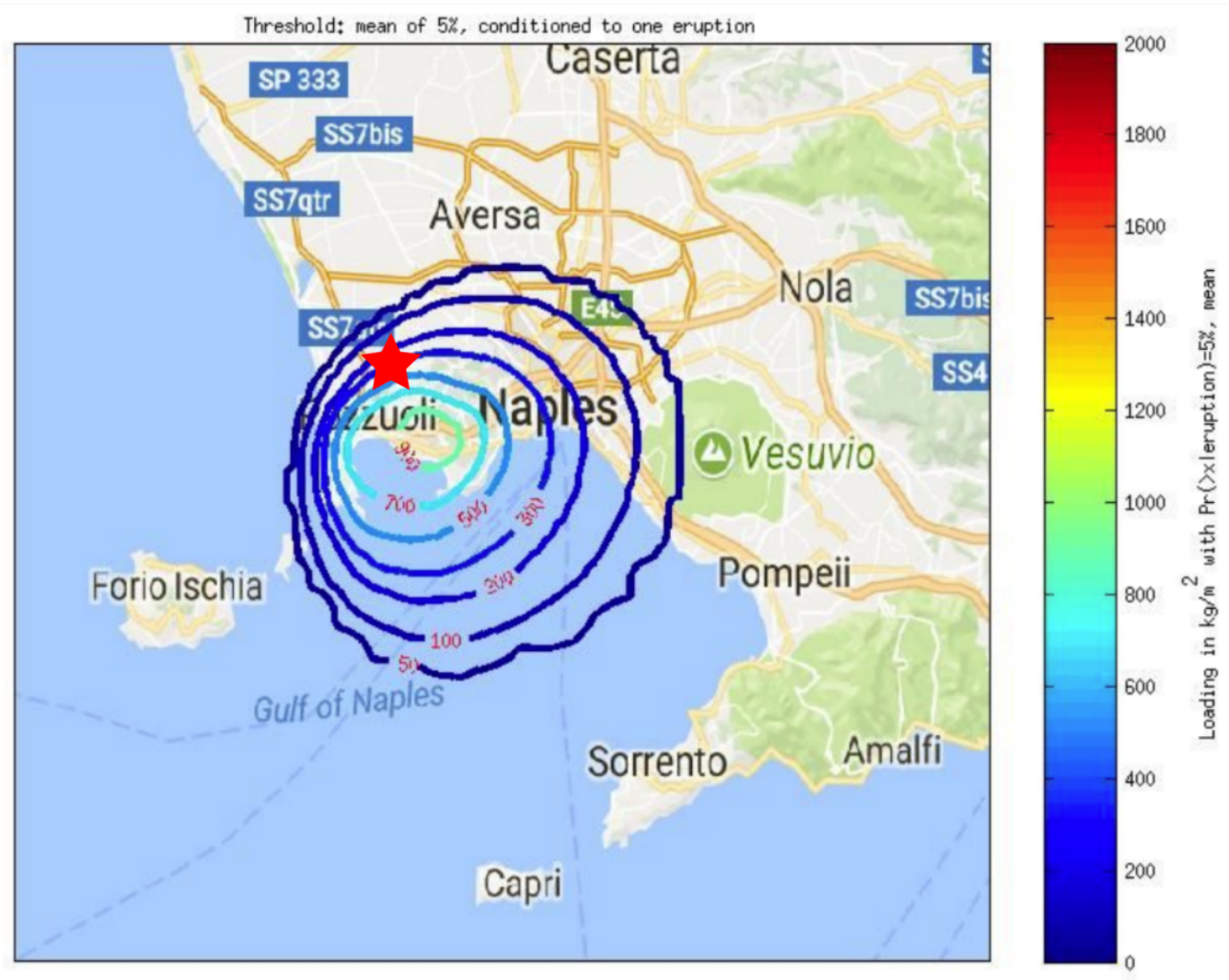

Figure 2: The location reference site for this study (red star) along with the hazard map relative the probability of $5 \%$ of exceedance, conditional upon the occurrence of one eruption of whatever size and from whatever vent at Campi Flegrei (mean of the epistemic uncertainty). The figure has been obtained modifying the Figure 11 of the corrigendum to the paper Selva et al., 2018 (available at doi.org/10.1016/j.jvolgeores.2018.07.008); the original maps have been obtained with the MATLAB software. 
https://doi.org/10.5194/nhess-2021-213

Preprint. Discussion started: 23 July 2021

(c) Author(s) 2021. CC BY 4.0 License.

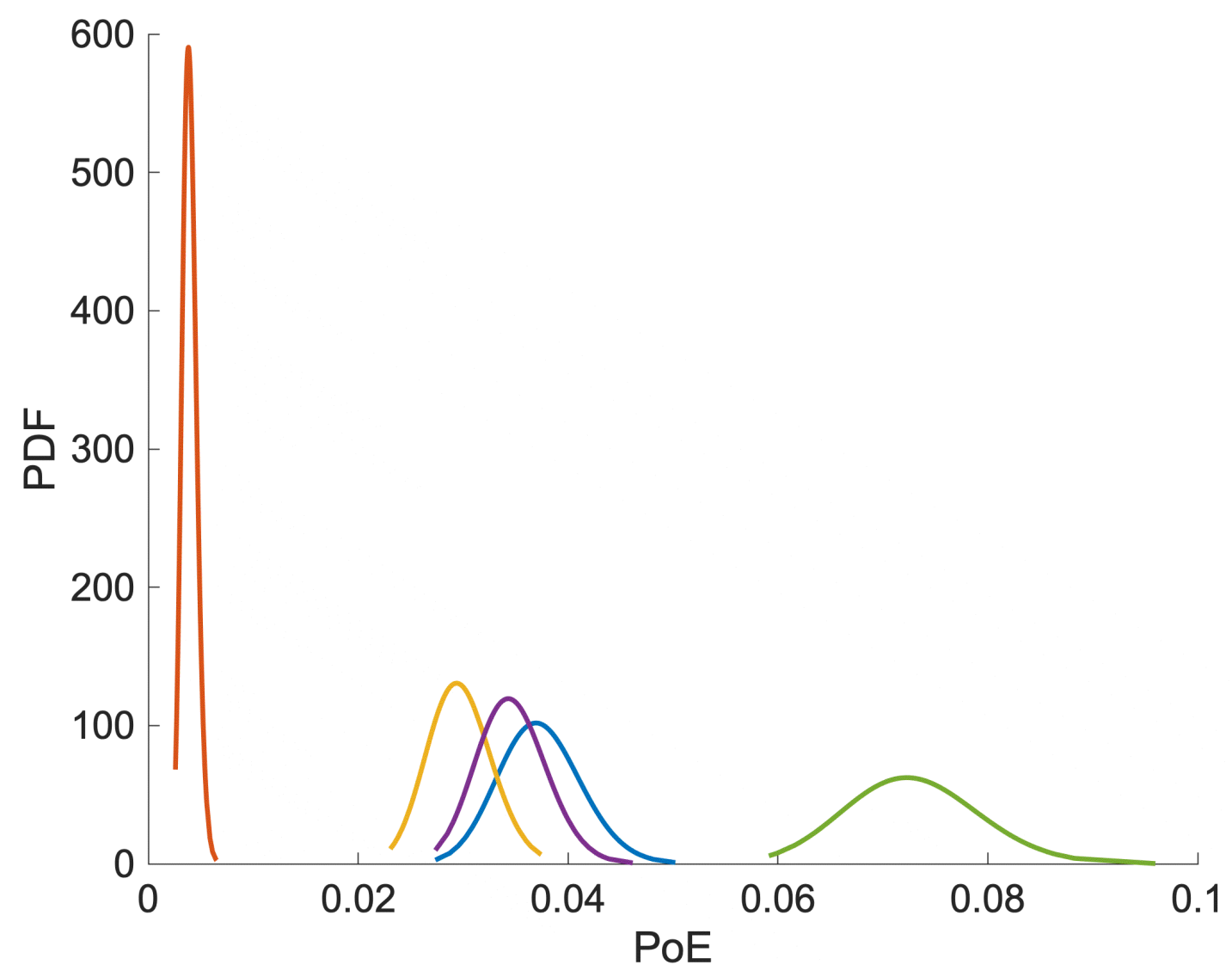

355 Figure 3: EED of each BET configuration relative to a tephra load threshold of $300 \mathrm{~kg} / \mathrm{m}^{2}$ for the site in Figure 2. 
https://doi.org/10.5194/nhess-2021-213

Preprint. Discussion started: 23 July 2021

(c) Author(s) 2021. CC BY 4.0 License.

(c) (1)

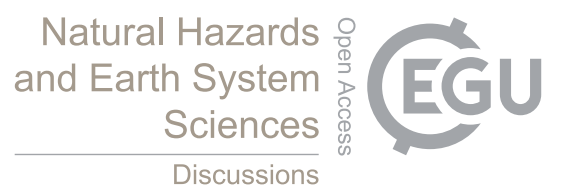

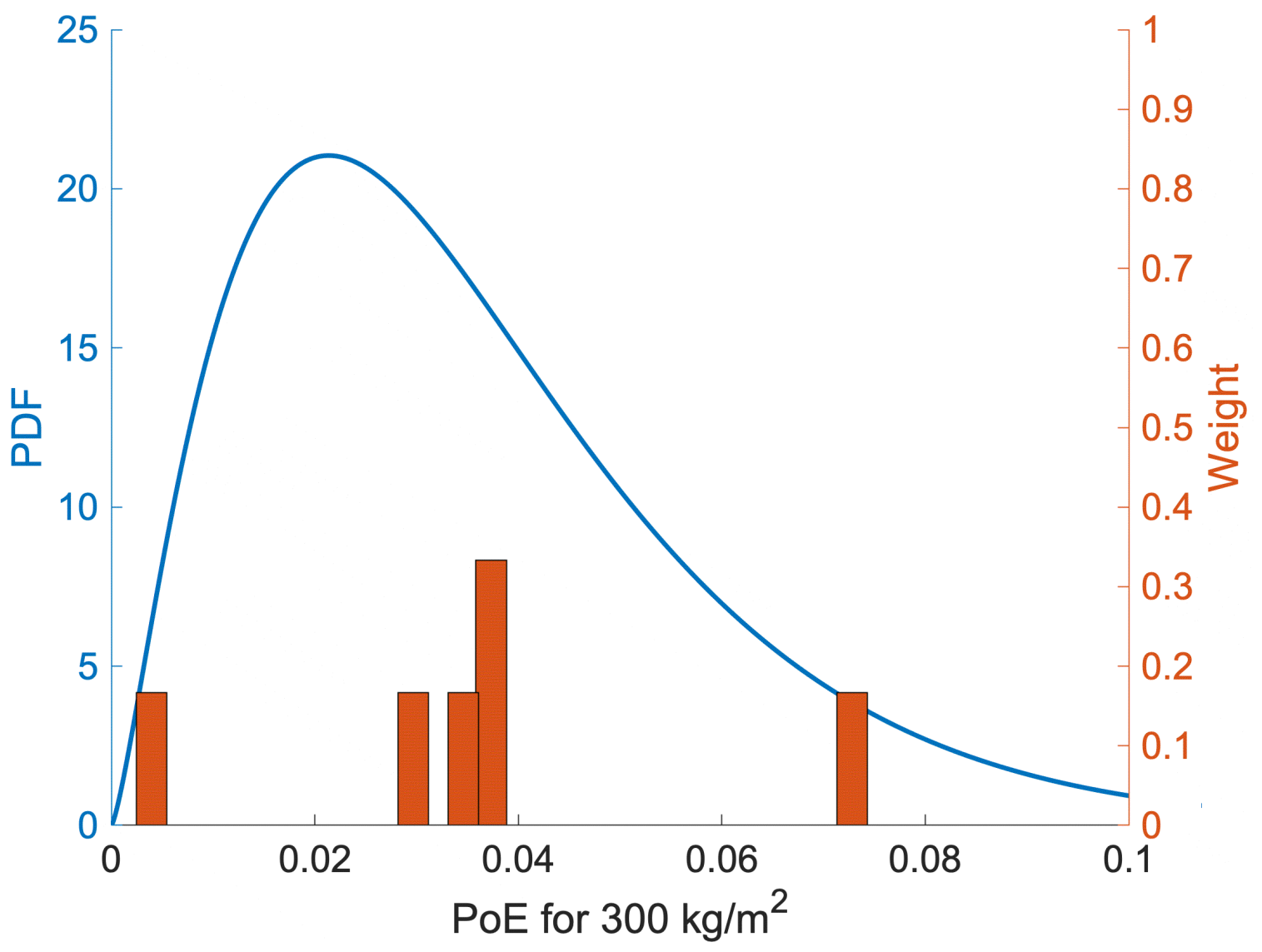

360 Figure 4: The overall EED obtained from the average of the five distributions of Figure 3. The EED is a Beta distribution with parameters given by equations 2 and 3. The histogram (right $y$-axis) shows the weight of each one of the five distributions. 